\section{HSE}

Historia Social y de la Educación

Social and Education History
Hipatia Press

www.hipatiapress.com

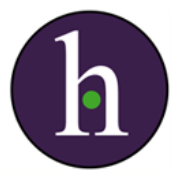

Instructions for authors, subscriptions and further details:

http://hse.hipatiapress.com

\title{
La Influencia sobre los Estudiantes del Profesorado Crítico del Tardofranquismo: el Caso de las Ciencias Sociales
}

Carlos Fuertes Muñoz ${ }^{1}$

1) Universidad de Valencia (España)

Date of publication: June $23^{\text {rd }}, 2016$

Edition period: June 2016 - October 2016

To cite this article: Fuertes, C. (2016). La Influencia sobre los Estudiantes del Profesorado Crítico del Tardofranquismo: el Caso de las Ciencias Sociales. Social and Education History 5(2), 188-194. doi:10.17583/hse.2016.1996

To link this article: http://dx.doi.org/10.17583/hse.2016.1996

PLEASE SCROLL DOWN FOR ARTICLE

The terms and conditions of use are related to the Open Journal System and to Creative Commons Attribution License (CC-BY). 


\section{The Influence of the Critic Teacher of the late "Franquismo" on Students: the Case of Social Sciences}

Carlos Fuertes Muñoz ${ }^{1}$

Universidad de Valencia (Spain)

\section{Abstract}

In this text we aim to reflect on the progress of critical attitudes between the teachers during the last stage of Franco dictatorship, analyzing various factors that favored its important influence on the critical awareness of students, an issue scarcely studied. On the basis of an analysis focused in Valencia of testimonies of students and teachers, the perspective adopted favors the exposure of concrete examples that will allow us, with qualitative and microhistorical metodologies, a deeper understanding of the particular critical influence of teachers of social sciences and humanities teachers. Overall, this study highlights the importance in the change of student attitudes in the late Francoism, not only on factors such as socio-cultural changes, greater contact with the outside or the daily visibility of meetings, demonstrations and repression, but also of the fundamental critical influence of these teachers.

Key words: francoism, social history, teachers, history of education, didactics of social sciences. 


\section{La Influencia sobre los Estudiantes del Profesorado Crítico del}

\section{Tardofranquismo: el Caso de las Ciencias Sociales}

Carlos Fuertes Muñoz

Universidad de Valencia (España)

\section{Resumen}

En este texto pretendemos reflexionar sobre el avance de las actitudes sociales críticas entre el profesorado durante la última etapa de dictadura franquista, analizando diversos factores que favorecieron su importante influencia en la concienciación crítica de los estudiantes, una cuestión escasamente estudiada. A partir de un análisis de testimonios de estudiantes y profesores centrado en Valencia, la perspectiva adoptada privilegia la exposición de ejemplos concretos que nos permitirán, desde metodologías cualitativas próximas a la microhistoria, comprender con mayor profundidad la particular influencia crítica del profesorado de ciencias sociales y humanidades. En conjunto, este trabajo pone de manifiesto la importancia en el cambio de actitudes de la juventud estudiante del tardofranquismo, no solo de factores como los cambios socioculturales, el mayor contacto con el exterior o la visibilización cotidiana de las asambleas, las manifestaciones y la represión, sino también de la fundamental influencia crítica de estos sectores del profesorado.

Palabras clave: franquismo, historia social, profesores, historia de la educación, didáctica de las ciencias sociales 


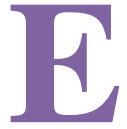

n este trabajo reflexionaremos sobre la influencia crítica sobre los estudiantes ejercida por el profesorado crítica del tardofranquismo, focalizando nuestra mirada en el caso de las ciencias sociales y en ejemplos procedentes de la realidad educativa valenciana. En un primer apartado, dedicado al estado de la cuestión y el marco teórico-metodológico, planteamos a grandes rasgos el cambio de actitudes de sectores del profesorado, exponiendo la perspectiva adoptada, el contexto más amplio de la investigación, las fuentes y la metodología utilizadas. Los siguientes dos apartados pretenden facilitar una primera aproximación a la particular influencia crítica del profesorado de ciencias sociales y humanidades; cuestión que ha sido constatada a través de nuestra investigación pero escasamente estudiada y sistematizada con anterioridad, de ahí el carácter más descriptivo de estos dos apartados. Así, expondremos diversos ejemplos concretos que, de acuerdo con una perspectiva cualitativa y microhistórica (Levi, 2003), nos permitirán visibilizar y comprender con detalle la particular influencia crítica de este colectivo docente, la cual será analizada diferenciando entre el más movilizado, conflictivo y conocido ámbito universitario, en el segundo apartado, y la más masificada, apaciguada y desconocida enseñanza media, en el tercero. En el cuarto apartado, de tipo más analítico, reflexionaremos sobre los diversos factores que, a la luz de nuestro análisis, pudieron favorecer la influencia crítica de dichos sectores del profesorado sobre el alumnado, cuestión que, en última instancia, contribuyó a minar la legitimidad de la dictadura franquista entre las nuevas generaciones.

\section{El Cambio de Actitudes del Profesorado en el Tardofranquismo y su Influencia Crítica: Marco General de la Investigación}

La relación entre las ciencias de la educación y la historiografía sobre las actitudes sociales bajo la dictadura franquista ha sido hasta la fecha, lamentablemente, escasa, de lo que se deriva la escasa integración de las cuestiones relacionadas con el sistema educativo en el debate sobre el "consenso" y el "disenso" durante el régimen de Franco (Del Arco, Fuertes, Hernández y Marco, 2013). Así, si bien es cierto que contamos con 
abundantes trabajos que han analizado a través de fuentes como las leyes o los contenidos de los libros de texto el papel del sistema educativo en las estrategias de legitimación del franquismo (Castillejo, 2008; Fernández Soria, 1998; Valls, 1983; Valls Montes, 1984, 2009), seguimos careciendo, pese a algunas excepciones más bien recientes, de estudios atentos a cuestiones tan relevantes como la recepción del proyecto educativo oficial (Cruz, 2001; Fuertes, 2016a), la realidad cotidiana de las aulas o las actitudes que realmente desarrollaron estudiantes (Fuertes, 2014) y profesores (Gómez, 2016; Groves, 2012, 2013).

Partiendo de esta constatación, en este estudio atenderemos al avance de las actitudes sociales críticas entre el profesorado durante la última etapa de la dictadura franquista. En efecto, uno de los fenómenos más destacables del sistema educativo durante las últimas dos décadas del franquismo es el creciente cambio de actitudes sociopolíticas y pedagógicas entre minoritarios aunque influyentes sectores del profesorado de los distintos niveles y espacios, incluyento los centros religiosos. Un colectivo entre el cual los discursos críticos con la realidad sociopolítica, las prácticas docentes alternativas y las protestas por la mejora de sus condiciones laborales se extendieron de manera destacada, todo ello en un contexto, en el que, en medio del predominio de los profesores situados en posiciones que podríamos denominar como "afranquistas" o "conformistas" pero poco activamente entusiastas del régimen, se asistió a un claro descenso del profesorado "ultra", así como, en conjunto, a una atenuación de las actitudes más reservadas, atemorizadas y sumisas entre el profesorado (Gil, Pérez y Sánchez, 2012; Gómez, 2009; 2016; Groves, 2013; Molina, 2011; Lázaro, 2008).

Diversos factores contribuyen a explicar dicha transformación, debiendo destacar particularmente la llegada a la edad adulta de unas nuevas generaciones de profesores escasamente marcadas por el trauma de la guerra y expuestas a nuevas experiencias de socialización en parroquias, clubs juveniles o centros de trabajo, así como en los propios institutos, facultades o escuelas de Magisterio dónde ellos mismos se formaron previamente. Este cambio de actitudes entre sectores del profesorado, siendo en sí mismo significativo de la erosión de los pilares institucionales y socializadores del régimen, revistió aún mayor importancia en tanto en cuanto los enseñantes más implicados en la renovación pedagógica, las reivindicaciones laborales 
y la lucha por el cambio democrático, contribuyeron, en realimentación con el cambio de actitudes de amplios sectores de los estudiantes (Martín, González y Ortiz, 2009; Rodríguez Tejada, 2009), tanto a agudizar el fracaso del proyecto educativo oficial, como a la conversión de no pocas aulas y centros educativos en espacios de socialización alternativa y aprendizajes democráticos de la juventud, actuando asimismo como agentes de dinamización sociocultural de los barrios y pueblos del tardofranquismo (Groves, 2011).

Teniendo en cuenta la escasez de estudios atentos a la influencia del profesorado crítico sobre los estudiantes, en este trabajo trataremos de aproximarnos a esta concreta dimensión a partir de un análisis basado en testimonios de profesores y alumnos, en el cual nos centraremos en la incidencia del profesorado de ciencias sociales y humanidades de la enseñanza media y universitaria, colectivo sobre el que a penas existen investigaciones monográficas y que, sin embargo, a la luz de nuestra investigación sobre Valencia, ejerció una particularmente relevante influencia sociopolítica alternativa a los valores oficiales. Inspirándonos en aquellas perspectivas que desde las áreas de la Historia de la Educación (Agulló, 2004, 2007; Brock, 2009; Cruz, 2001; Mayordomo, Agulló y García, 2013) y la Didáctica de las Ciencias Sociales (Beltrán, Martínez y Souto, 2006; Llácer, 2007; Parra, 2013) tratan de aproximarse con metodologías cualitativas a los fenómenos de la difusión y la recepción de la enseñanza de contenidos sociales, culturales y políticos, reflexionaremos a partir de diversos ejemplos sobre la intensidad, los factores que la favorecieron y las formas que asumió dicha influencia crítica del profesorado.

El origen de este trabajo cabe ubicarlo en una extensa investigación sobre las actitudes sociales ante y durante la dictadura franquista relacionadas con temáticas tan diversas como la memoria de la guerra civil, la situación económica o el sistema educativo, las cuales fueron estudiadas mediante fuentes muy diversas, aunque priorizando el uso de fuentes orales y memorialísticas como vía de aproximación a la vida cotidiana y las actitudes sociopolíticas de la "gente corriente" (Fuertes, 2016b). Así, se recurrió a la realización por parte del autor de 77 entrevistas semiestructuradas en profundidad a personas de diversas edad, sexo, perfil sociolaboral e implicación política, seleccionadas en Valencia y su área 
metropolitana a través de diversas vías que trataron de garantizar la representatividad de la muestra y sobre cuya metodología, sometida a crítica en su fase inicial por parte de especialistas de reconocido prestigio en la historia oral, me he detenido con mayor detalle en diversos trabajos anteriores (Fuertes, 2010a, 2010b; Fuertes y Gómez, 2011). Junto a dichas entrevistas, se recopilaron y analizaron diversos relatos autobiográficos recogidos en libros de memorias, recopilaciones de testimonios y trabajos de otros autores, la mayoría de los cuales con una pretensión más divulgativa que científica; una práctica que sin duda presenta particularidades y problemáticas específicas que conviene tener muy en cuenta, pero que, al tiempo y con las debidas precauciones, puede aportar materiales y perspectivas complementarias, de ahí que cada vez sea más frecuente (Cabana, 2007; Figes, 2009; Halbmayr, 2010).

El análisis de contenido de los diversos testimonios se llevó a cabo mediante la elaboración de "reportajes narrativizados" (Lejeune, 1989), en los que tratamos de escribir una especie de "comentario de texto" o reseña de cada informante, así como con la organización de diversos ficheros temáticos de citas en torno a objetivos prioritarios de la investigación, que nos permitieron clasificar los distintos resultados en diversas categorías, tipologías y temáticas. De todos estos testimonios construidos y rastreados en el marco de dicha investigación, en los siguientes apartados escogeremos algunos ejemplos de estudiantes y profesores que nos serán de especial utilidad para ilustrar la especial influencia crítica del profesorado valenciano de ciencias sociales y humanidades de la enseñanza media y universitaria, cuestión que será contextualizada, asimismo, utilizando la bibliografía especializada existente ${ }^{2}$.

\section{La Influencia del Profesorado Crítico en las Facultades de Filosofía y Letras, Derecho y Económicas}

Para empezar, en las facultades universitarias valencianas parece detectarse una particular influencia crítica del profesorado de humanidades y ciencias sociales. Dolores Sánchez Durá y Pascual Masiá, destacan el notable ambiente de apoyo a los cambios democráticos entre el claustro de la Facultad de Filosofía y Letras durante los años sesenta y setenta, donde destacan nombres como Carlos Paris, Jose Luis Pinillas, Manuel Garrido, 
Joan Reglà, Miquel Tarradell o Emili Giralt, o incluso el que llegara a ser decano, Miquel Dolç, quién sin llegar a simpatizar tanto con la lucha del movimiento estudiantil, buscó la negociación antes que el enfrentamiento (Sánchez Durá y Masiá, 2000, p. 275). Fernanda Mancebo (1999, p. 148152), que estudió a finales de los cincuenta y primeros de los sesenta, destaca cómo a pesar de la continuidad de actitudes conformistas y adaptativas entre buena parte del profesorado -"el miedo sellaba los labios de muchos de nuestros maestros y profesores"-, "tuve la suerte de entrar en una Facultad de Filosfía y Letras (que por entonces era de lo mejor de España) que iba despertando", destacando la influencia crítica de profesores como Reglá, Jover, Tarradell o Dolç. Jaime Millás, recuerda que la influencia de varios de esos profesores fue clave en el inicio de su concienciación que le acabaría llevando no a militar en organizaciones políticas pero sí a implicarse en movimientos cristianos de base y en las iniciativas culturales de la sociedad Studio S.A., tan importantes en la transformación en un sentido "alternativo" de la vida cultural -y politica- de la capital del Turia. "Cuando ingreso en la Facultad de Filosofia y Letras descubro allí una mina de humanistas (...) Eso fue un acicate para que se produjese en mí un proceso de transformación ideológica y un modo distinto de concebir la vida" (Marí y Zabala, 1999, p. 76-77).

También en la Facultad de Derecho crecieron progresivamente los profesores críticos. Rafael evoca, al igual que otros licenciados en Derecho valencianos (Zabala, 1994, p. 62), el cambio de ambiente desde mediados de los cincuenta. Así, recuerda cómo en sus años de estudiante, entre 1953 y 1958, en un ambiente caracterizado, más allá de la defensa a ultranza del régimen por parte del catedrático falangista Diego Sevilla, por unos profesores "por supuesto conservadores todos", aunque no destacados por su adhesión inquebrantable al franquismo, más bien por ser "apolíticos", el profesor Miaja suponía un contraste, "tampoco lo exteriorizaba, aunque no era igual que los demás" (Entrevista a Rafael F.). Este último profesor, nacido en 1908, de familia republicana y represaliado por el régimen franquista, pasaría cinco años en la cárcel, entre 1936 y 1941, y sólo sería finalmente rehabilitado para la docencia universitaria en los años cincuenta, cuando llegaría a la UV como catedrático de Derecho Internacional, dónde muchos alumnos inquietos de Derecho admirarían sus clases y rumorearían sobre sus antecedentes políticos y penales, llegando a ser decano a finales de 
los sesenta (VVAA, 1981). Por otra parte, Rafael destaca cómo, animados por el profesor Murillo, impulsor del desarrollo de la sociología en España durante el franquismo, realizaron trabajos sobre la constitución republicana de 1931 o comparaciones con sistemas políticos democráticos. Más allá de Valencia, Juanjo, proveniente de una familia extremeña consentidora con el franquismo, estudió Derecho en la Universdad de Salamanca a finales de los cincuenta y primeros sesenta y destaca que en el despertar de su conciencia crítica fueron importantes, además del rechazo de la represión policial y del control ejercido por el SEU, las clases de Enrique Tierno Galván: “A mí me cogió en segundo de carrera cuando acababa de ser indultado junto con Aranguren y García Calvo (...) Sus clases eran muy interesantes. Totalmente, Tierno sí, Tierno era muy crítico, pero no trataba de influirte, daba los materiales para que tú te informaras" (Entrevista a Juanjo M.).

La reconstruccion de Vicente Cuñat (1999), profesor en la Facultad de Derecho de la UV, presenta un ambiente de finales de los sesenta y principios de los setenta marcado por el crecimiento de los profesores críticos. Enrique, estudiante de Derecho entre 1968 y 1973 que no participó en protestas pero si llegó a concienciarse durante aquellos años de la necesidad de un cambio democrático, recuerda como importante en este sentido la influencia de Manuel Broseta, junto a las relaciones personales con otros profesores y compañeros (Entrevista a Enrique D.M.). Ricardo, estudiante también en esa última etapa de la dictadura y activista integrado en el PCE, recuerda que la influencia que ejercían los profesores demócratas se veía reforzada por el hecho de que estos suponían un contraste llamativo en medio de un contexto dominado por profesores "carcas" (Entrevista a Ricardo P.). José Antonio Noguera, implicado en el incipiente movimiento estudiantil, destaca igualmente el impacto que les generaba el acercamiento, a traves de estos profesores críticos y las lecturas que les recomendaban, a la realidad constitucional de los países democráticos (Marí y Zabala, 1999, p. 73-74).

Carmen Alborch, estudiante de derecho entre 1965 y 1970 y también implicada en el movimiento estudiantil, afirma que "tuvimos suerte, tuvimos muy buenos profesores en mi generación" y destaca la influencia que en su formación política ejercieron profesores como Adolfo Miaja de la Muela, el líder del PSP en Valencia Manuel Sánchez Ayuso o Manuel Broseta, decano a principios de los setenta que destacó por impedir que la policía entrara en 
la Facultad y defendió con frecuencia en la prensa valenciana un nuevo modelo de universidad democrática. Este último fue el que más influyó en Carmen Alborch, quién lo define como "un demócrata de perfiles singulares" que "nos mostró un modelo de enseñanza en la que se podía opinar y que te invitaba al dialogo y la reflexión". Tras acabar sus estudios, ella misma pasaría a trabajar como profesora de Derecho, implicándose en la lucha por la democracia pese a las amenazas que recibieron ella y muchos otros profesores, quiénes, destaca, "llamábamos la atención", apuntando asimismo al hecho de que "una buena parte de los mejores profesores de la universidad de entonces se decantaron claramente contra la dictadura y una buena parte de los mejores estudiantes, también. Hubo esa confluencia y fue muy importante" (Alborch, 2009, p. 41-52).

Respecto al ambiente de los profesores en la Facultad de Derecho de la UV, Lola recuerda el contraste que se producía entre clases como las de Derecho Canónico, dónde el profesor, "quan explicave los imperativos del matrimonio: "Las mujeres que se salgan de la clase porque esto es muy escabroso", y clases como las de Miaja de la Muela, las cuales eran "gracioses" aunque "dins d'uns límits", o las de dos catedráticos jóvenes, Díez Picazo y Córdoba (Entrevista a Lola Monferrer). Josep-Lluís inició su compromiso político en la Facultad de Derecho durante los años sesenta, implicándose en el movimiento estudiantil y más tarde en la defensa de antifranquistas represaliados por el TOP. Así, en su relato destaca cómo a fin de entender el proceso de concienciación crítica de cada vez más estudiantes, conviene tener en cuenta que "a Dret hi havia un moviment a nivell de professorat molt bo, perquè també era el moment de despuntar els demòcrates", cuestión que "potenciava" el movimiento estudiantil, "perquè allò de negar l'entrada als grisos o d'autoritzar assemblees, tot això creava un consciència política molt ràpida, molt ràpida..." (Entrevista a Josep-Lluís A.).

Respecto a la Facultad de Económicas, creada el curso 1967-1968, pronto se convirtió en un hervidero de jóvenes profesores no numerarios entre los que abundaron los demócratas, particularmente los vinculados al socialismo. Así, jóvenes profesores como Ernest Lluch, Vicent Soler, Manuel Sanchez Ayuso, Victor Fuentes o Emèrit Bono, contribuyeron a extender un ambiente crítico en la Facultad. Sanchez Ayuso, primer decano elegido democráticamente de la Facultad de Económicas y Empresariales, "permitió 
que las aulas permaneciesen siempre abiertas, e impidió siempre que pudo que la policía campase a sus anchas" (Alborch, 2009, p. 42). Toni, estudiante de económicas entre 1971 y 1976, considera cómo, si bien no llegó a militar en organizaciones políticas ni a implicarse muy activamente en el movimiento estudiantil, la influencia de algunos de estos profesores fue clave, junto a la visibilización cotidiana de las asambleas, las manifestaciones y la represión, en el desarrollo de su conciencia antifranquista y favorable al cambio democrático, para alguien que venía de una familia conservadora, con un padre camisa vieja falangista, y que en los Maristas dónde hizo el bachillerato siguió manteniendo actitudes conformistas. Destacando la influencia de profesores como Sánchez Ayuso, Bono o Lluch a través de clases y conversaciones informales en el día a día de sus estudios, concluye: "O siga que, jo pense que totes eixes influencies d'eixes persones, junt a que lo que estàs estudiant, jo pense que és lo que ha decantat que jo tinga una idea diferent a lo que mon pare" (Entrevista a Toni G.).

\section{La Influencia Crítica de los Profesores de Humanidades y Ciencias Sociales de la Enseñanza Media}

Más allá de las facultades, numerosos testimonios de estudiantes y profesores de la época nos permiten reconstruir la particular influencia sociopolítica del profesorado crítico y demócrata de ciencias sociales y humanidades en la enseñanza media. Varios testimonios destacan, para empezar, la influencia de profesores pertenecientes a la generación de preguerra. Rafael, que estudió a principios de los cincuenta en la conocida Academia Castellano de Valencia, recuerda que no se vivía una intensa presión religiosa, afirma que "aquel ambiente me marcó" destacando particularmente que "a mi me marcó muchó” José Bueno Ortuño, licenciado en Letras, profesor de Historia y de Literatura, represaliado, militante del PCE antes de la guerra, redactor de "Nueva Cultura" y amigo de Alberto García Esteve, Juan Gil Albert y Juan Renau, entre otros. Su influencia favoreció, entre otras cosas, que Rafael eligiera estudiar Derecho, acabando convirtiéndose en uno de los abogados más implicados en la defensa de los antifranquistas valencianos (Entrevista a Rafael F.). Bastantes años después, en la segunda mitad de los sesenta, Vicente Vergara también pasó por aquel 
centro, destacando igualmente que, con la base de "esas preocupaciones políticas que has escuchado en casa", con un padre antiguo militante de la Derecha Regional Valenciana decepcionado con el régimen por la represión falangista de posguerra, "a mí me influyó muchísimo" el paso por la Academia Castellano y el contacto con profesores represaliados por la dictadura. Al igual que Rafael, destaca la particular influencia de las clases de Literatura y de Historia de José Bueno, las cuales "me llevaron a preocuparme mucho" por cuestiones sociopolíticas y por el pasado republicano, pues "te hablaba de García Lorca, te hablaba de Miguel Hernández, de Joan Fuster", concluyendo que "todo eso es una base que va haciendo que tomes un poco de conciencia de que estás viviendo bajo una dictadura y que hacen falta unas libertades democráticas" (Gómez y Sánchez, 2009, p. 295).

También Vicent Álvarez destaca cómo en el instituto de Xàtiva a finales de los cincuenta "empezamos a tener el apoyo de algunos profesores" de Literatura que "nos ayudaron un poco a tomar algo más de conciencia". Así, señala cómo allí "se leía a Lorca, a Machado, que no se podía leer en ningún lado, se hablaba de la Guerra Civil, y de ese tipo de cosas". Asimismo, destaca cómo el profesor de Francés contribuía también a romper esquemas socioculturales y sociolingüísticos al aprovechar determinados momentos de sus clases para hablarles en valenciano y sobre cuestiones de la cultura valenciana. Por otra parte, señala que "además, teníamos el contrapunto de la Formación del Espíritu Nacional" (FEN), apuntando en este sentido al modo en que el contraste entre asignaturas y profesores afectaba negativamente a FEN y, por el contrario, facilitaba la mayor receptividad hacia otras materias y profesores, por contraste con el discurso sociopolítico e histórico allí recibido. En conjunto, considera que, junto a las relaciones establecidas con varios compañeros estudiantes, las influencias de estos profesores, "sobre todo las lecturas, porque leer a gente de la Generación del 98, de la del 27, todo eso, de alguna manera, fomenta el espíritu crítico", favorecieron que cuando acabaron el bachillerato "salimos del instituto con una posición ya muy claramente antifranquista" (Gómez y Sánchez, 2009, p. 173).

El testimonio de Julio, catedrático de Geografía e Historia en varios institutos de Valencia desde la segunda mitad de los años sesenta, es bien ilustrativo del avance de los márgenes de acción del profesorado de ciencias sociales y humanidades, así como de sus preocupaciones pedagógicas, en los 
últimos años de la dictadura. Este profesor e investigador en Historia, discípulo de Jover, aunque estuvo condicionado hasta el final de la dictadura por la represión sufrida por su familia en la posguerra, critica las tradicionales visiones monolíticas y estereotipadas de la enseñanza bajo el franquismo como genéricamente autoritaria, basada en la disciplina extrema y sin ningún atisbo de innovación, pluralidad metodológica o márgenes de acción para el profesorado: "se ha creado un poco de cliché sobre la enseñanza aquella, que eso se refleja a veces en las películas (...) como si estuviéramos en el siglo XIX”. Frente a ello, considera que dichos márgenes existían y más aún en los últimos años, cuando asimismo "hubo una preocupación pedagógica creciente" tratando de ir más allá de la clase magistral y de introducir "enseñanzas activas". Grupos que fueron surgiendo “espontáneamente, creo yo", y a los que se incorporó el propio Julio, miembro del Grupo Edetania y que a su vez fue profesor-tutor de los jóvenes recién licenciados que debieron empezar a realizar un período de prácticas y de formación pedagógica como requísito necesario para poder acceder al cuerpo de profesores de enseñanza media.

Su testimonio es particularmente interesante respecto a la cuestión de los márgenes de acción del profesorado de Historia en la enseñanza media para la introducción de discursos críticos en la elaboración de materiales didácticos y en la dinámica de las aulas. Así, Julio destaca, por ejemplo, cómo en una obra colectiva para la asignatura de Historia de España de $6^{\circ}$ de Bachillerato se le encargó la parte sobre la Guerra Civil, "y ahí se me planteó un problema de objetividad histórica y creo que no quedé insatisfecho en ese sentido porque pude también no hacer ni leyenda negra ni leyenda rosa". Así, destaca que siendo como era "un tema difícil" trató de hacer un esfuerzo de objetividad "respecto a las causas, respecto al desarrollo y también con una adaptación de comprensión para alumnos del bachillerato de la época, preuniversitarios, alumnos de 16 años...", señalando en conjunto "que se pudo hacer, vamos, yo lo pude hacer y no quedé insatisfecho ni he tenido críticas de ninguna clase hablando de las causas". Preguntado por si su papel como profesor de Historia bajo la dictadura le creaba algún tipo de conflicto moral o de sentimiento de mala conciencia por estar pudiendo contribuir al éxito del sistema de adoctrinamiento en los valores del franquismo, Julio pone en valor con claridad los márgenes de acción de que disponía, afirmando: "Lo he sentido pero he procurado 
contrarrestarlo... porque si yo daba una materia cuya intención la veía clara... procuraba plantearla de otra manera que era la más adaptada a mis ideales". Así, en una muy extensa pero esclarecedora cita pone el ejemplo de como solía tratar en el PREU un tema que venía en el currículum sobre "historia de los concilios", permitiendo asimismo apreciar el avance de las actitudes críticas y la pérdida del miedo entre las nuevas generaciones de estudiantes y como ello a su vez favorecía el uso crítico de los mencionados márgenes de acción:

Pues yo me lo planteé a mi manera, que era un estudio de la época. En alguna ocasión efectivamente me surgieron algunas preguntas de las que tú en realidad... me acuerdo concretamente que hubo un caso de hablar de las luchas en estos primeros tiempos del cristianismo entre los nósticos, los maniqueos, las primeras herejías hasta el concilio de Éfeso, de Antioquía, y todo esto. Y hubo, porque a veces las reacciones de los alumnos eran muy inteligentes, muchas veces te preguntaban cosas que te hacían pensar: "¿Por qué se enfrentaban, por disputas de... si el sexo de los ángeles?” Tiene efectivamente una base real (...) muchas herejías de estas (...) había grupos de estos que seguían que eran grupos sociales y al mismo tiempo religiosos, tenían estas ideas, yo tenía que explicarlo porque además luego se examinaban. Un chico me dijo, "Oiga... ¿y no sería que detrás de estas ideas habían unos intereses materiales?". Y digo: "Si, pues probablemente, efectivamente...". Y entonces había que ver si estas ideas respondían... yo procuraba seguirle, ¿no? (...) Y eso es lo que había que hacer aunque muchas veces no se expresara claramente, pero podía hacerlo, y lo digo porque muchas veces (...) este es un ejemplo concreto (...) te suministraban una serie de temas que a ellos les parecía, afectos a su ideología pero que yo procuraba pues eso... No podía tener una crisis de conciencia por integrarme en el sistema educativo porque no existía otro y tenía que subsistir (Entrevista a Julio S.).

El testimonio de Fernanda Mancebo, socializada en la cultura alternativa y el antifranquismo en la Facultad de Filosofía y Letras de Valencia y profesora de Historia, resulta de gran interés para reconstruir sus estrategias docentes y más en general el ambiente existente en el instituto de Benetússer, aún entonces filial del Luis Vives de Valencia, en el que empezó a trabajar en 1963. En dicho centro, a pesar de que el ambiente no era tan 
"fácil" como en las facultades o los institutos "de la capital", Fernanda destaca como "hasta nosotros llegaban los ecos del movimiento estudiantil" y de la "lucha obrera" que se fraguaba en los propios barrios y polígonos industriales de la zona. Allí sintonizó con varios profesores con "inquietudes" y "problemas" compartidos, todos ellos preocupados por cambiar la manera de enseñar en línea con los postulados de la renovación pedagógica, fomentando la participación del alumnado y con ello la formación de ciudadanos responsables y autónomos, así como involucrándose en protestas laborales y favoreciendo también la movilización de los estudiantes por sus propias problemáticas. Igualmente, destaca cómo una de las tareas que con mayor satisfacción vivió fue la de intentar estimular el deseo de seguir estudiando en los hijos de la clase trabajadora que poblaba las aulas de este centro del área metropolitana de Valencia, señalando cómo, de hecho, muchos de los universitarios más contestatarios y activos del tardofranquismo fueron antiguos alumnos suyos, "aquellos hijos/as de trabajadores que llegaron a las facultades con gran esfuerzo, becas, trabajando, radicalizados y algunos vinculados a los partidos o grupúsculos más a la izquierda: el PC m-l, el FRAP, Bandera Roja, trotkistas... Se nos iban de las manos...".

En su testimonio resulta especialmente interesante su reflexión sobre como la asignatura de Historia, "aunque fuera en el bachiller hasta cuarto que se impartía en las filiales, daba el juego suficiente para plantearme mi "compromiso", aunque ello le supuso enfrentamientos con el director del centro, también profesor de Historia, el jefe de estudios y el cura. Así, destaca cómo aunque nunca llegó a militar en ningún partido, "yo y muchos como yo teníamos claro cual era el frente de nuestra lucha, desde una formación cristiana y liberal: sobre todo nuestro trabajo bien hecho". Así, destaca cómo "la ruptura conscioente con el sistema educativo vigente era nuestra meta" y cómo "nuestra tarea consistía en posibilitar esa funesta manía de pensar que inmediatamente llevaba a algunos a la crítica y al descontento", señalando cómo "respecto a los contenidos era fácil denostar a Felipe II y entonar la loa de la Ilustración", mientras que al tiempo "empezamos a introducir la pedagogía activa, la posibilidad de que los alumnos participasen y se expresasen libremente en las clases en grupo, los debates y las excursiones" (Mancebo, 1999, pp. 154-155). 
Mercedes Madrid, licenciada en Filología Clásica por la Universdad de Salamanca, dónde tomó contacto con el movimiento estudiantil, empezó a ejercer como profesora de Griego en el Instituto Juan de Garay de Valencia desde 1970, integrándose en el PCE y asumiendo tareas de responsabilidad en la numerosa sección de enseñanza media del partido en la provincia. $\mathrm{Su}$ testimonio aporta claves muy interesantes que profundizan en algunas de las ideas expuestas por Julio o Fernanda Mancebo, añadiendo asimismo nuevos matices. Por un lado, al igual que Fernanda, Mercedes destaca con rotundidad como, a diferencia de algunos compañeros profesores antifranquistas que dejaron en un segundo plano su tarea docente para centrarse en la política "en mayúsculas", ella siempre priorizó la enseñanza considerando que su trabajo era político porque ejercía un papel clave de concienciación de los estudiantes y de motivación para potenciar el acceso a la universidad de los hijos de la clase trabajadora, frente al profundo clasisimo del sistema educativo: "Nosotros pensábamos que la lucha contra Franco tenía que ser sacar el mayor número posible de gente bien educada, con espíritu crítico, con un nivel alto de conocimientos, y para nosotros ésa era la lucha. O sea que la lucha pasó por mi profesión (...) nuestra política era nuestro trabajo". Asimismo, en la línea de Julio, enfatiza la importancia de "una cosa buena que había por aquellas fechas, y que siempre estuvo", que "fue algo que se llamó la libertad de cátedra, que yo no entiendo porque el franquismo defendió eso", destacando que "yo creo que eso es lo que permitió que mucha gente dentro de clase se explayara a gusto y que, en fin, se pudiera...", pues "eso fue una cosa que se respetaba mucho, o sea, que tú en clase podías..." y "la gente se cortaba poco", aunque al tiempo destaca que la inspección educativa le mostró sus reticencias "porque trabajaba en grupos y que eso podía ser subversivo, y una mala práctica", una metodología que seguían varios profesores del centro y gracias a la cual "conseguíamos un rendimiento grande que de otra manera no hubiera sido posible".

En consonancia con los anteriores testimonios, destaca cómo utilizó la enseñanza de la historia de la antigua Grecia para generar actitudes críticas sobre la realidad sociopolítica, como "por ejemplo, lo hacía explicando el libro II de Tucídides, que es donde Pericles habla de lo que es la democracia, y eso lo explicaba en clase, lo tenía en el programa", así como "haciendo un seminario de presocráticos para explicar el materialismo dialéctico, que era 
para introducir el marxismo, que era lo que en ese momento pensábamos que era el método de entender la historia, la teoría...". Destaca asimismo Mercedes cómo durante el famoso Proceso de Burgos de 1970, hubo un grupo de profesores, que una vez a la semana lo trataron con los alumnos en forma de seminarios a partir de la lectura del semanario de sucesos El Caso que era utilizado como una fuente legal que seguía con detalle el juicio para, a partir de la misma, generar interrogantes y cuestionar la represión franquista. "Me pareció de una valentía tremenda en ese momento que se difundiera, y que los alumnos, sobre todo los mayores, supieran lo que estaba pasando con el Proceso de Burgos, que a mí me parecía muy importante y que había un silencio total sobre ello".

En opinión de Mercedes, la labor sociopolítica del profesorado crítico en su instituto fue eficaz en un contexto de receptividad del alumnado destacando cómo, junto a alumnos "diríamos casi sin concienciar" y a una casi total ausencia de "fachas", "había grupos muy activos" que incluso "ya estaban vinculados con partidos", por lo que "entonces lo que había era mucha complicidad (...) había muchísima complicidad, muchísima". "Entonces era muy grato dar clase porque mirabas a los ojos y sabías perfectamente los que te habían captado y los que no te habían captado. O sea, parte de la clase... Muchas veces había muchos mensajes, diríamos: el que tenía el oído abierto lo captaba perfectamente y sabía por dónde iban las cosas". Asimismo, señalaba como en su opinión, su capacidad para conectar con los estudiantes guardaba relación con las actividadas encaminadas a dinamizar la vida sociopolítica y cultural del centro más allá del horario lectivo, destacando igualmente como la clave última de su eficacia en la concienciación crítica estaba en el hecho de que, mediante métodos didácticos innovadores y temas silenciados, intentaban enseñar a pensar críticamente pero huyendo de lo que sería una "propaganda" o "adoctrinamiento" simplista:

Yo desde luego en ningún momento me autocensuré en clase. Cierto es que yo nunca utilicé la clase para hacer una propaganda descarada. Yo pensaba que parte de nuestra, de mi misión pedagógica, es que tenía que darles a los chicos y a las chicas armas para que ellos reflexionaran y vieran realmente, como era lógico, que la dictadura era un sistema 
perverso de gobernarse, y luego ya que cada cual pensara lo que quisiera (Gómez y Sánchez, 2009, pp. 238-245)

En el instituto Cid Campeador, el avance de la innovación en la enseñanza de la Historia y la Geografía en los últimos años de la dictadura y los primeros de la transición, que afectó tanto a los contenidos como a la metodología y los fundamentos explicativos, fue impulsado por aquellos profesores más vinculados al antifranquismo y a la defensa del cambio democrático, los cuales entendieron que su labor docente debía jugar un papel fundamental precisamente en dicho proceso, de cara a la conformación de un alumnado crítico y social y políticamente comprometido (Llácer, 2013, pp. 471-472). Conxa Delgado (2013), recuerda cómo en un instituto Benlliure caracterizado por la escasez de profesores abiertamente adictos y el predominio de profesores conformistas y acríticos, fue especialmente enriquecedora y sorprendente la influencia del joven profesor de FEN, Pere Enguix, así como de las profesoras de Literatura y Francés, quiénes "van començar a fer-me veure que el món de l'educació també volia un canvi en el nostre país, i que no sols els i les estudiants estàvem per eixa feina”. Así, destaca cómo la profesora de Literatura les habló de Antonio Machado, mientras que la de Francés, "quan teníem necessitat de fer una assemblea a l'aula, ella se n'eixia al corredor per vigilar que no vinguera cap conserge a controlar què és el que passava". Los alumnos y alumnas del Isabel de Villena también tienden a destacar las influencias críticas de los profesores de Humanidades. Así, dos de ellas recuerdan cómo el profesor de Francés les ponía canciones de Brel y Moustaki "que hablaban de libertad y cambios individuales", destacando otra cómo la profesora de Literatura les recitaba "los versos de León Felipe, poeta perseguido por el régimen franquista que utilizaba su poesía como un arma social”. Igualmente, la profesora de Filosofía de este centro evoca cómo intentó desarrollar nuevas formas de enseñanza que promovieran la concienciación crítica del alumnado, rompiendo, al igual que muchos otros jóvenes profesores de esta materia, el habitual esquema cronológico en el tratamiento de la historia de la Filosofía, dedicándose en cambio a alternar autores contemporáneos con otros de otras épocas e introduciendo problemas sociales y culturales propios del mundo actual en el tratamiento del pensamiento filosófico (Cueca y Martínez Bonafé, 2011, p. 221-223). 
Conviene destacar, en último lugar, cómo también en los centros religiosos se apreció la influencia del profesorado crítico de humanidades y ciencias sociales. Así lo recuerdan, por ejemplo, antiguos alumnos del colegio de Jesuitas en Valencia (Marí y Zabala, 1999, p. 77), dónde en relación con los cambios generales de la Iglesia y con los particulares cambios de esta congregación, se asistió a una particularmente intensa penetración de los discursos críticos de la mano de profesores como el historiador y jesuita Ramiro Reig, que impartía clases en las Escuelas Profesionales y acabó implicándose el mismo como activista sindical en el movimiento obrero valenciano (Gómez y Sánchez, 2009, p. 265). Ricardo, que estudió en La Salle de Palma de Mallorca a finales de los sesenta y primeros setenta, recuerda el fuerte contraste producido entre el profesor de FEN y otros profesores con un discurso mucho más abierto y crítico: "aquel hombre era el colmo ya del cinismo y de... ¡En un contexto en el que ya todo eso estaba ya de vuelta y media! Pues en paralelo a ese profesor teníamos otros que nos hablaban ya muy abiertamente de muchas cuestiones". Destaca particularmente el caso del profesor de Historia, un laico con un estilo de vida bastante peculiar y moderno, de quién recuerda que, sin llegar a tratar en ningún momento el siglo $\mathrm{XX}$, dedicó gran espacio en su materia al tratamiento de la revolución industrial, haciéndoles leer a Tuñón de Lara para tratar el movimiento obrero, del mismo modo que a Ubieto y a Jover, dos de los profesores universitarios más renovadores de la historiorografía española bajo el franquismo (Entrevista a Ricardo J.F.).

\section{Factores y Formas en la Influencia del Profesorado Crítico}

Tal y como hemos podido apreciar mediante los ejemplos analizados, el constatado cambio de actitudes de ciertos sectores del profesorado durante los últimos años de la dictadura franquista, tuvo sin duda, por minoritario que fuese este fenómeno, una repercusión notable sobre el alumnado, debiendo tener en en cuenta que este colectivo tenía, por las particularidades de la profesión docente, un público o "audiencia" considerable, más aún en el contexto de expansión de la enseñanza básica, media y universitaria que en aquellos años se vivió (Canales, 2005). En este sentido, la contextualización de los ejemplos expuestos en el marco más amplio de 
nuestra investigación nos permite apreciar una cuestión que, entendemos, no se ha subrayado lo suficiente. Esto es, cómo, junto a muchos otros factores como los cambios socioculturales, el mayor contacto con el exterior o la visibilización cotidiana de las asambleas, las manifestaciones y la represión, en el cambio de actitudes de la juventud estudiante del tardofranquismo fue fundamental la influencia crítica de estos sectores del profesorado.

En efecto, entendemos que estos profesores, tanto mediante el ejemplo de la participación en protestas laborales o el sufrimiento de la represión, como a través de los discursos transmitidos y las experiencias generadas en clases, actividades extracurriculares o conversaciones informales, contribuyeron a reforzar la concienciación crítica de sectores no desdeñables de los estudiantes, que fueron asi generándose nuevos interrogantes, familiarizándose con prácticas y llevando a cabo aprendizajes de valores que les distanciaban de la cultura oficial. Tal y como muestran numerosos testimonios de profesores y alumnos manejados en nuestra investigación, algunos de los cuales hemos podido apreciar en los anteriores apartados, diversos factores pudieron favorecer esta influencia. Factores como el prestigio social y autoridad moral de los profesores de la época; la conexión generacional que favorecía la juventud de muchos de los profesores críticos -a pesar de que también hemos apreciado la influencia de la represaliada generación de preguerra-; su apoyo a los estudiantes frente a la represión; su "rareza" en positivo, tanto a nivel de discurso sociopolítico crítico como a menudo también a nivel estético, actitudinal y didáctico, con una mayor cercanía y una metodología más dinámica y participativa o, entre otros, su hábil utilización de los "márgenes de acción" con los que contaban para la difusión de prácticas alternativas y discursos críticos, incluso en una dictadura (Cueca y Martínez, 2011, p. 221-223; Entrevistas a: Rosa J.; Rafael F.; Julio; Ricardo J.F; Gómez y Sánchez, 2009, p.137 y pp. 237-245; Mancebo, 1999, pp. 154-155; Mayordomo, Agulló y García, 2013, p. 479487; Serra, 2009, p. 203; Vallés, 2000, p. 151-156).

Otro factor importante que contribuyó a reforzar las complicidades del profesorado crítico con el alumnado fue su implicación en la realización de diversas actividades extraescolares y extracurriculares que fomentaron el aprendizaje de valores críticos y la identificación con referentes culturales alternativos y a menudo silenciados o perseguidos por la dictadura. En el caso de los centros de primaria y enseñanza media del País Valenciano, fue 
fundamental, por una parte, la progresiva introducción de la enseñanza del valenciano, uno de los buques insignia de muchos de los incipientes grupos de enseñantes preocupados por la transformación del sistema educativo franquista, tanto mediante su utilización informal y puntual en clases impartidas en castellano, como forma de resistencia individual y ruptura de los esquemas establecidos, como a través de la realización de actividades extraescolares optativas (Vallés, 2000, p. 152-153; Mayordomo, Agulló y García, 2007, p. 163-180, 252-254 y 262). Junto a las clases de valenciano, conviene destacar la realización en no pocos colegios e institutos bajo el impulso de este sector crítico del profesorado de otras actividades que conectaban con las inquietudes del alumnado al tiempo que a menudo difundían referentes alternativos a los oficiales, tales como grupos de teatro, cine-fórums, concursos literarios, periódicos murales, revistas, conocimiento del País Valenciano, guitarra, ajedrez, tenis o audiciones musicales (Cueca y Martínez, 2011, p. 223-225; Mayordomo, Agulló y García, 2007, p. 163 164; Llácer, 2013).

Igualmente, las charlas de sexualidad, un "tabú grande" en opinión de Mercedes Madrid, que impulsó con "un éxito rotundo" una de ellas en el instituto Juan de Garay de Valencia (Gómez y Sánchez, 2009, p. 243), se convirtieron en un claro desafío a los patrones culturales del nacionalcatolicismo, siendo recordadas hoy por las alumnas del Isabel de Villena "com una experiència que els obria camins de llibertat que no havien somiat les seues mares" (Cueca y Martínez, 2011, p. 223). Paco, joven maestro que descubrió la importancia de la educación sexual de la mano de un instructor "distinto a los demás" en los campamentos de la OJE para alumnos de Magisterio, intentó introducirla en sus clases de últimos cursos de primaria en el centro privado Luis Vives de Paterna durante el tardofranquismo y la transición, junto a otras innovaciones pedagógicas que si bien le causaron conflictos cotidianos con la dirección del centro, parece que al tiempo no tuvieron una mala acogida entre estudiantes y familias: "Pero yo recibía las broncas bien, primero, porque sabía que lo que hacía y yo creo que lo hacía bien, no hacía nada del otro mundo, y nunca un padre o una madre me dijo nada, 'Oye que te estás pasando', o 'Ese tema mejor no', entonces... (...) Y nos íbamos por ahí, 'Alé a la huerta, pues a estudiar las cosas a la huerta (...) a dibujar la torre [de Paterna] (...) a Valencia a ver no 
se qué'. Y eras así, porque te gustaba y disfrutabas y los críos disfrutaban y punto" (Entrevista a Paco M.).

Aunque la renovación metodológica y los discursos críticos se extendieron entre sectores muy diversos del profesorado, las fuentes manejadas en nuestra investigación sobre Valencia permiten apreciar una particular influencia sociopolítica alternativa a los valores oficiales de los profesores de muy distintas materias de ciencias sociales y humanidades de la enseñanza media y universitaria, constatable en centros de muy diverso tipo y perfil, que abarcan desde facultades como Filosofía y Letras, Derecho y Económicas, hasta institutos públicos de barrios y localidades muy variadas, academias privadas, escuelas profesionales o centros religiosos. De este modo, podríamos decir que la dictadura se enfrentaba a la existencia de crecientes fisuras en un pilar fundamental de sus estrategias de adoctrinamiento y legitimación a través del sistema educativo, teniendo en cuenta que los profesores de estas materias estaban destinados a jugar un papel clave en la socialización de las nuevas generaciones en los valores políticos y referentes culturales oficiales. Desde luego, no se trata de negar que en el marco de materias como Biología o Educación Física no se pudiesen llevar a cabo aprendizajes de prácticas y valores que contribuían a fomentar el pensamiento autónomo y crítico, así como a acrecentar el distanciamiento de la juventud respecto a la dictadura. Pero sí de subrayar que materias como Historia, Economía, Literatura, Derecho o Filosofía, por la centralidad de los contenidos culturales, sociales y políticos, facilitaban enormemente el uso sutil de los mencionados márgenes de acción del profesorado para favorecer el pensamiento crítico del alumnado respecto a la España del tardofranquismo. Unos estrechos aunque no despreciables márgenes que, aunque aunque no excluyesen la vigilancia y la represión, en aras de la "libertad de cátedra" y protegidos por el tradicional individualismo docente, podían permitir llevar a cabo adaptaciones en el uso de la programación didáctica, los materiales o los libros de texto, con capacidad igualmente para orientar la lectura del alumnado hacía determinados énfasis o matices que solo aparecían entrelíneas en los textos analizados.

Sobre las formas y particularidades de la influencia crítica del profesorado de ciencias sociales y humanidades dan buena cuenta los ejemplos descritos en los anteriores apartados. Tratando de sistematizar, podríamos decir que, huyendo de lo que podríamos considerar un 
adoctrinamiento antifranquista burdo y simplista, los profesores de ciencias sociales y humanidades fueron especialmente hábiles en el análisis sutilmente crítico de temáticas aparentemente inocuas y que, de hecho, a menudo estaban destinadas a jugar un papel central en la transmisión de las narrativas histórico-culturales oficiales. Buenos ejemplos de ello serían la contrastación entre la visión crítica de la época de Felipe II y la valoración positiva de la Ilustración que, aprovechando las ventajas del pasado más remoto para hacer juicios políticos, servía a Fernanda Mancebo (1999, p. 154-155) para criticar los sistemas políticos autoritarios o subrayar la importancia de las libertades individuales. $O$ el tratamiento que Julio realizaba de la historia de las concilios, en el que, sustituyendo las tradicionales visiones positivistas e historicistas, apostaba por un cambio en los fundamentos explicativos hacia posturas cercanas la historia social y política, apuntando a la importancia en la dinámica socio-histórica del conflicto sociocultural, las desigualdades y los intereses contrapuestos (Entrevista a Julio S.).

Junto a ello, el profesorado crítico de ciencias sociales y humanidades se distinguió por su apuesta, no exenta de riesgos, por la introducción en las aulas de temáticas mucho más silenciadas y especialmente oportunas para la estimulación del pensamiento crítico del alumnado y la difusión de referentes culturales alternativos a los de la dictadura. Hemos apreciado varios ejemplos relativos a cómo diversos profesores de lengua y literatura acercaron a sus alumnos a la poesía y la narrativa de las generaciones del 98 y del 27 o a la lengua y la cultura valencianas (Cueca y Martínez Bonafé, 2011, pp. 221-223; Gómez y Sánchez, 2009, p. 173). Profesores de la Facultad de Derecho como el sociólogo Murillo promovieron la comparación de la realidad jurídico-política franquista con la de otros sistemas políticos democráticos, como la propia Segunda República Española (Entrevista a Rafael F.; Marí y Zabala, 1999, p. 73-74). A menudo estas temáticas silenciadas fueron tratadas con habilidad mediante la utilización de fuentes legales, tal como hacía Mercedes Madrid con su reflexión sobre el concepto de democracia a partir del libro II de Tucídides (Gómez y Sánchez, 2009, p. 242). Esta cuestión se vio favorecida, asimismo, por la reducción de la censura en los últimos diez años de la dictadura, la cual, aunque muy limitada, fue especialmente apreciable en publicaciones académicas, cuya calidad y perspectiva crítica aumentó en un contexto de 
renovación de las humanidades y las ciencias sociales, como ejemplifican, en el ámbito de la historiografía, el libro sobre la guerra civil en el que participó Julio o la difusión de libros como los de los prestigiosos historiadores universitarios Ubieto, Jover o Tuñón de Lara (Entrevistas a Julio S. y Ricardo J.F.).

Junto a la cuestión de las temáticas y las perspectivas con que estas eran tratados, a menudo los profesores de ciencias sociales y humanidades enseñaron a pensar críticamente mediante la utilización de métodos didácticos innovadores. Conviene destacar, en este sentido, la apuesta por un modelo de enseñanza participativa, activa y dialógica, en la que, como destacaba una antigua alumna del profesor de Derecho Manuel Broseta se incitaba al alumnado a opinar y debatir (Alborch, 2009, p. 44). En consonancia con este planteamiento general encajan la difusión de los trabajos en grupo, las excursiones o la realización de seminarios de lectura, como el mencionado relativo al "Proceso de Burgos" a partir de un análisis de el semanario El Caso, en todo un ejemplo de original uso de una fuente legal para el tratamiento de una temática tan silenciada en las aulas y los medios como era la represión franquista y la propia existencia de una activa oposición (Gómez y Sánchez, 2009, p. 244). Otro ejemplo de esta innovación docente es el de la ruptura en el tradicional esquema cronológico en la enseñanza de la historia de la filosofía llevada a cabo por una profesora del Isabel de Villena, estrategia que iba encaminada asimismo a la introducción en sus clases de problemáticas sociales y culturales contemporáneas. En este mismo centro cabe destacar la utilización de la música como recurso didáctico por parte de algunos profesores, las cuales introducían, asimismo, cuestiones como las libertades individuales o el problema de la represión durante los últimos compases de la dictadura, como destacan varios alumnos a propósito de la escucha en clase de cantautores como los fanceses Brel y Moustaki o de la canción de Lluís Llach dedicada a los obreros asesinados por la policía en Vitoria en 1976 (Cueca y Martínez Bonafé, 2011, p. 221-223).

La innovación en la selección y enfoque de los temas así como en las estrategias didácticas podía ver reforzada su influencia por el hecho de que estos profesores de ciencias sociales y humanidades suponían un notable contraste en un panorama dominada por un profesorado apolítico y conservador, como recuerdan antiguos alumnos de la Facultad de Derecho 
de Valencia (Entrevistas a Rafael F., Ricardo P. y Lola M.). En relación con ello, conviene destacar cómo la influencia crítica de este sector del profesorado más vinculado al antifranquismo y la lucha por la democracia, suponía suponía una notable amenaza para la dictadura, en tanto en cuanto los encargados de impartir las materias de ciencias sociales y humanidades constituían un pilar fundamental de sus estrategias de legitimación entre las nuevas generaciones. Todo ello en un contexto en el que, de hecho, la utilización política por parte del régimen de los contenidos socioculturales transmitidos mediante materias como Historia o FEN se enfrentaba cada vez más a numerosas dificultades y a un escaso entusiasmo entre el alumnado (Boyd, 1997, p. 223-255; Cruz, 2001, p. 237-239; Fuertes, 2016a). Una cuestión que guarda relación, entre otros factores, con el creciente cambio de actitudes entre las nuevas generaciones de estudiantes del tardofranquismo, la cual se tradujo en una creciente demanda de nuevas respuestas e informaciones sobre cuestiones socioculturales e históricas que favoreció una considerable complicidad y receptividad hacia los sectores más críticos del profesorado (Entrevista a Julio S.). Una cuestión, en fin, sobre la que aun queda mucha por decir, siendo necesario que un trabajo introductorio como este sea completado por análisis mucho más sistematizados atentos a los diversos factores y formas de la influencia crítica del profesorado crítico del tardofranquismo, valorando igualmente su intensidad, sus límites y sus resistencias. De todo ello esperamos ocuparnos en futuras investigaciones.

\section{Notas}

${ }^{1} \mathrm{El}$ autor participa en el Proyecto de Investigación "Derechas y nación en la España contemporánea. Culturas e identidades en conflicto”, ref. 2014-53042-P, del Ministerio de Economía y Competitividad

${ }^{2}$ Se citará por el nombre de pila y se indicará la inicial del primer apellido, de acuerdo con la voluntad de los informantes. 
190 Fuertes- Influencia del profesorado crítico del tardofranquismo

\section{Referencias}

Agulló, M.C. (2004). Entre la retòrica i la realitat: Juventudes de la Sección Femenina. València (1945-1975). Educació i Història: Revista d'Història de l'Educació, 7, 247-272. Recuperado de http://roderic.uv.es/handle/10550/11205

Agulló, M.C. (2007). Possibilitats i riscs de les fonts orals en la investigació historicoeducativa. Educació i Història: Revista d'Història de l'Educació, 910, 27-39. Recuperado de

http://www.raco.cat/index.php/EducacioHistoria/article/view/223113

Albroch, C. (2009). La ciudad y la vida. Barcelona: RBA.

Beltrán, J., Martínez, N., y Souto, X.M. (2006). Los profesores de historia y la enseñanza de la historia en España. Una investigación a partir de los recuerdos de los alumnos. Enseñanza de las ciencias sociales: revista de investigación, 5, 55-69. Recuperado de http://www.raco.cat/index.php/EnsenanzaCS/article/view/126317

Boyd, C.P. (1997). Historia Patria. Política, historia e identidad nacional en España, 1875-1975. Barcelona: Pomares.

Brock, A. (2009). Producing the 'socialist personality'? Socialisation, education, and the emergence of new patterns of behaviour. En M. Fullbrook (ed.). Power and society in the GDR, 1961-1979: the "normalisation of rule?" (pp. 220-252). New York : Berghahn Books.

Cabana, A. (2007). Entre a resistencia e a adaptación: a sociedade rural galega no franquismo (1936-1960). Universidade de Santiago de Compostela, Tesis doctoral.

Canales, A.F. (2005). La expansión de la enseñanza media en la España del desarrollismo y la transición. En: Actes del Congrés La Transició de la dictadura franquista a la democràcia (pp.393-401). Barcelona: UABCEFID.

Castillejo, E. (2008). Mito, legitimación y violencia simbólica en los manuales escolares de Historia del franquismo (1936-1975). Madrid: Universidad Nacional de Educación a Distancia.

Cruz, J.I. (2001). El yunque azul. Frente de Juventudes y sistema educativo: razones de un fracaso. Madrid: Alianza Editorial.

Cueca, M. y Martínez, A. (2011). Anar a l'institut als anys setanta: L'INB Isabel de Villena de València a la fi del franquisme. En A. Mayordomo, M.C. 
Agulló y G. García (coords.). El patrimoni historicoeducatiu valencià (pp. 207-225). Valencia: UV-CEIC Alfons El Vell.

Cuñat, V. (1999). Los decanos demócratas: Manuel Broseta. En B.Sanz y R. Bello (eds.). Memoria del antfiranquismo. La Universidad de Valencia bajo el franquismo (pp. 261-275). Valencia: UV.

Del Arco, M.A., Fuertes, C., Hernández, C., y Marco, J. (eds.) (2013). No sólo miedo. Actitudes políticas y opinión popular bajo la dictadura franquista (1936-1977). Granada: Editorial Comares.

Delgado, C. (2013). Curs 72-73, COU a l'IES Benlliure. En A. Mayordomo, M.C. Agulló y G. García (coords.). Instituts i batxillers (pp. 471-474). Valencia: UV-CEIC Alfons El Vell.

Fernández Soria, J.M. (1998). Educación, socialización y legitimación política: España, 1931-1970. Valencia: Tirant lo Blanch.

Figes, O. (2009). Los que susurran: la represión en la Rusia de Stalin. Barcelona: Edhasa.

Fuertes, C. (2010a). El problema del consenso en el franquismo (c.1957c.1976). Reflexiones sobre el estudio de las actitudes sociopolíticas de los españoles. En M. C. Fuentes, J. Contreras y P. López (eds.). II Encuentro de Jóvenes Investigadores en Historia Contemporánea. Granada: Universidad de Granada.

Fuertes, C. (2010b). La gente corriente ante la dictadura de Franco. Un proyecto de historia oral en Valencia. 16th International Oral History Association Conference, "Between Past and Future: Oral History, Memory and Meaning” (Praga, 7-11 Julio 2010).

Fuertes, C. (2011). Actitudes políticas de las clases populares durante el desarrollismo. Un estudio local de historia oral en Paterna (Valencia). En A. Cabana, D. Lanero y V. Santidrán (eds.). VII Encuentro de Investigadores sobre el Franquismo (pp. 368-379). Santiago de Compostela: Fundación 10 de Marzo.

Fuertes, C. (2014). Vida cotidiana, educación y aprendizajes políticos de la sociedad española durante el franquismo. En. M.P. Ledesma e I. Saz (eds.). Historia de las culturas políticas contemporáneas en España y América Latina. Tomo IV. Del franquismo a la democracia, 1936-2013 (pp.53-79). Madrid: Marcial Pons-Prensas Universitarias de Zaragoza. 
192 Fuertes- Influencia del profesorado crítico del tardofranquismo

Fuertes, C. (2016a). La recepción de la educación política oficial bajo el franquismo: el caso de la Formación del Espíritu Nacional. IX Encuentro de Investigadores del Franquismo. 80 años de inicio del franquismo (Granada, 10 y 11 de marzo de 2016): Granada, Comares (en prensa).

Fuertes, C. (2016b). Legitimación, educación y actitudes sociales bajo la dictadura franquista (Valencia, c.1950-.c.1975). Universidad de Valencia, Tesis doctoral inédita.

Fuertes, C., y Gómez, A. (2011). El Tribunal de Orden Público en el País Valenciano. Testimonios de la represión política y el antifranquismo, Valencia, FEIS.

Gil, G., Pérez, M., y Sánchez, D. (2012). Antifranquismo y renovación pedagógica. El Seminario de Pedagogía del Colegio de Doctores y Licenciados del D.U. Valencia (1966-1978). Valencia: FEIS.

Gómez, A., y Sáchez, D. (eds.) (2009). jAbajo la dictadura! Tres generaciones de antifranquistas en el País Valenciano. Valencia: FEIS.

Gómez, F. (2009) El PCE y las movilizaciones en la enseñanza (1965-1982). En R. Quirosa-Cheyrouze, L.C. Navarro y M. Fernández (coords.). Congreso Internacional Historia de la Transición en España. Las organizaciones políticas (pp. 553-568). Almería: Instituto de Estudios Almerienses-Estudios del Tiempo Presente.

Gómez, F. (2016). El magisterio contra Franco. De la resistencia a la acción colectiva (1965-1978). Universidad de Alicante, Tesis doctoral inédita.

Groves, T. (2011). El maestro rural como agente de cultura alternativa durante la transición española: el caso de la provincia de Salamanca. Aula: Revista de Pedagogía de la Universidad de Salamanca, 17, 133-143. Recuperado de http://revistas.usal.es/index.php/0214-3402/article/viewFile/8399/9109

Groves, T. (2012). Everyday struggles against Franco's authoritarian legacy: pedagogical social movements and democracy in Spain. Journal of Social History, 46(2), 305-334. doi: 10.1093/jsh/shs094

Groves, T. (2013). Teachers and the Struggle for Democracy in Spain, 19701985. Basingstoke: Palgrave Macmillan.

Halbmayr, B. (2010). Las dificultades de interpretar con métodos de Historia Oral. Historia, Antropología y Fuentes Orales, 43, 57-169. Recuperado de http://www.jstor.org/stable/25759021?seq=1 - page_scan_tab_contents Lázaro, L.M. (2008). El impulso crítico a la renovación pedagógica: de finales del franquismo a la transición democrática. En A. Mayordomo, M.C. Agulló 
y G. García (coords.). Canviar l'escola, canviar la societat: La renovació pedagògica valenciana al segle XX (pp. 65-127). Valencia: UV-CEIC Alfons El Vell.

Lejeune, P. (1989). Memoria, diálogo y escritura. Historia y Fuente Oral, 1, 3367. Recuperado de https://www.jstor.org/stable/27753228

Levi, G. (2003). Sobre microhistoria. En P. Burke (ed.). Formas de hacer historia (pp. 119-145). Madrid: Alianza Editorial.

Llácer, V. (2007). La enseñanza de la historia desde la perspectiva del profesorado de bachillerato. Didáctica de las ciencias experimentales y sociales, 21, 53-89. Recuperado de https://dialnet.unirioja.es/descarga/articulo/2475993.pdf

Llácer, V. (2013). Innovació didáctica i participació educativa en temps de canvi. El cas de l'Institut Cid Campeador de la ciutat de València (19701985). En A. Mayordomo, M.C. Agulló y G. García (coords.). Instituts $i$ batxillers (pp. 347-375). Valencia: UV-CEIC Alfons El Vell.

Mancebo, M.F. (1999). Hijos de un Dios menor. En B.Sanz y R. Bello (eds.). Memoria del antfiranquismo. La Universidad de Valencia bajo el franquismo (pp. 145-160). Valencia: UV.

Marí, R., y Zabala, F. (1999). La Valencia de los años 60. Valencia: Ayuntamiento de Valencia.

Martín, O., González, D. y Ortíz, M. (2009). 'Envenenando a nuestra juventud'. Cambio de actitudes y militancia juvenil durante el segundo franquismo. Historia Actual Online, 20, 19-33. Recuperado de https://dialnet.unirioja.es/descarga/articulo/3150133.pdf

Mayordomo, A.; Agulló, M.C., i García, G. (2007) Valencià a l'Escola: Memòria i testimoni. III Jornades d'Història de l'educació valenciana. València: UV-CEIC Alfons El Vell.

Mayordomo, A.; Agulló, M.C., i García, G. (coords.) (2013). Instituts $i$ batxillers, València: UV-CEIC Alfons El Vell.

Molina, M.D. (2011). La práctica viva y la experiencia colectiva de la renovación pedagógica. Historia de vida del MRP del País Valencià-Gonçal Anaya. Universidad de Valencia, Tesis doctoral inédita.

Parra, D. (2013). La conceptualización de la Historia escolar y sus implicaciones didácticas. Un estudio a partir del recuerdo de estudiantes de BUP. Didáctica de las ciencias experimentales y sociales, 27, 3-22. Recuperado de https://ojs.uv.es/index.php/dces/article/view/2653 
194 Fuertes- Influencia del profesorado crítico del tardofranquismo

Rodríguez, S. (2009). Zonas de libertad: dictadura franquista y movimiento estudiantil en la Universidad de Valencia. Valencia: PUV.

Sánchez Durá, D., y Masiá, P. (2000): Los movimientos estudiantiles. En M. Baldó (ed.). Historia de la Universidad de Valencia. Vol.III: La Universidad Liberal (siglos XIX-XX) (pp. 275-286).Valencia: UV.

Serra, X. (2009). Biografies parcials. Els 70 al País Valencià. Catarroja: Afers. Vallés, S. (2000). Josep-Lluís Bausset. Converses amb l'home subterrani.

València: Tàndem Edicions.

Valls, F. (1983). La enseñanza de la literatura en el franquismo (1936-1951).

Barcelona: Antoni Bosch.

Valls Montes, R.(1984). La interpretación de la Historia de España, y sus orígenes ideológicos, en el bachillerato franquista (1938-1953). Valencia: Instituto de Ciencias de la Educación-UV.

Valls Montes, R. (2009). Historia y memoria escolar: Segunda República, Guerra Civil y dictadura franquista en las aulas (1938-2008). Valencia: PUV.

VVAA (1981). In memoriam: Adolfo Miaja de la Muela. Revista Jurídica de la UNAM, XIII.

Zabala, F. (1994). La Valencia de los años 50. Valencia: Ayuntamiento de Valencia.

Carlos Fuertes Muñoz: profesor del Departamento de las Ciencias Experimentales y Ciencias Sociales de la Universidad de Valencia. Dirección de contacto: carlos.fuertes@uv.es 